\section{NUEVAS ESPECIES DE ESPACIOS}

\section{Javier Pérez-Herreras}

Profesor, Universidad de Zaragoza

La teoría de la capa límite de Prandtl tendría implicancias directas en arquitectura: la disposición y materialidad de los elementos construidos condicionarían la consistencia y calidad del aire (y del espacio) en torno a ellos. El espacio también es materia.

Palabras clave: Teoría de la arquitectura, tectónica, estructura, capa límite, Prandtl.

Prandtl's Boundary Layer Theory would affect the architectural notion of space: built elements (their materials and layout) do modify the consistency and quality of the surrounding air. Air, therefore space, is matter as well.

Keywords: Theory of architecture, tectonics, structure, boundary layer, Prandtl.
DEL AIRE DE NEWTON AL AIRE DE PRANDTL

$\mathbf{0}$ riente y Occidente han iniciado la búsqueda de un nuevo tipo de espacio que continúa con la intensa reciprocidad que hubo antes entre América y Europa en este mismo asunto. Dicha búsqueda se ha transformado, este último tiempo, en un nuevo laboratorio espacial. Si el laboratorio que unió América y Europa fue la patria doméstica, hoy es el del espacio público. En él ha surgido una nueva arquitectura, que se ha transformado con la ciudad y que ha convertido su habitación en un lugar abierto.

El renovado laboratorio ya no investiga sobre aquellos mecanismos de la modernidad que ligaban el espacio de su patria doméstica a un desconocido mundo. La vocación de exterioridad del espacio contemporáneo, convertido en lugar, hace de su anatomía el origen de una modificable materialidad. El logro de este nuevo espacio ganará, para su habitante, el viaje a una nueva patria. Un quimérico lugar, quizá desconocido a la casa que incorporó al hombre moderno a un nuevo tiempo, pero donde probablemente olvidó otros muchos sueños (fig. 1).

En 1851, el ingeniero Joseph Paxton resolvió el Palacio de Cristal como la construcción resultante de una repetición de elementos metálicos. Un esqueleto férreo, alámbrico y henchido de aire, dio vida a un nuevo lugar. Paxton diseñó una estructura tumbada sobre el suelo que nunca quiso ser arquitectura, pero que logró ser el alma del Palacio de Cristal. El aire que Paxton dibuja alrededor de su estructura en sus primeros croquis, es del mismo color y trazo que el aire de su interior. El aire previo y el aire final, a la estructura propuesta, son el mismo aire. No hay diferencias entre ellos. Es un aire de cualidad newtoniana, que exhibe una materialidad constante y ajena a cualquier posible acción externa. Hasta aquí, este espacio es aire, solo aire. Un gran vacío: "la nada", escribe Rochon (2010).

El éxito de Paxton fue mostrar este volumen de aire a través de su estructura metálica, convertida en su íntima alma. De la estructura emerge un aire visible. El alámbrico esqueleto se convierte en la anatomía de este primer y visible "especie de espacio". El resultado es un gran lugar, una nueva patria habitada por hombres y mujeres que buscan sueños que vienen de más lejos. Es obvio que el Palacio de Paxton no es la primera propuesta que une estructura y espacio, pero sí es la primera donde nosotros podemos ver claramente la íntima relación entre espacio, como aire visible, y su estructura, como esqueleto de aquel aire visible.

En 1904, el físico Ludwig Prandtl, un profesor de 29 años de la Technische Hochschule en Hannover, presentó en el Tercer
Congreso Internacional de Matemáticas de Heidelberg una nueva teoría que consideraba el aire como un fluido variable de diferentes capas: Boundary Layer. El hasta entonces aceptado concepto newtoniano de igual resistencia de un fluido fue reemplazado por una nueva concepción hidrodinámica (Prandtl, 1952). De acuerdo a esta, la resistencia del fluido resulta de las diferencias de presión e intensidad de fricción alrededor de un cuerpo. De tal manera, el aire es un material de viscosidad y agitación variable e identificable: máxima en su capa límite.

La nueva teoría revolucionó la forma de entender y analizar la dinámica de fluidos. A hombros de esta, la ingeniería aeronáutica desarrolló formas estructurales basadas en nuevas formas de cálculo. A la postre, nuevas capas límite que controlaban la viscosidad y agitación del aire alrededor de sus estructuras aeronáuticas (fig. 2).

La tesis de esta investigación sugiere que a la revolución aeronáutica que Prandtl provocó con su teoría de las capas límite ha seguido una revolución arquitectónica que se reconoce en aquellas nuevas arquitecturas públicas de Oriente. Sus estructuras dan habitación a otra modernidad, ahora líquida, señala Bauman. A aquellas nuevas estructuras aeronáuticas han seguido nuevas estructuras arquitectónicas. Anatomías para una distinta espacialidad arquitectónica, cuyo aire coagula en una nueva razón estructural. Con ellas, la estructura contemporánea se ha convertido en el origen de un nuevo espacio de manipulable densidad.

Aceptando este paralelismo, se pueden estudiar las diferentes materialidades y configuraciones del fluido-aire que forma esta nueva arquitectura, a través de la manipulación y conformación de sus capas límites, identificadas como su estructura. Es decir, se puede estudiar la materialidad del aire a través de la geometría y la posición de su estructura, como la originaria de su grado de viscosidad y finalmente de su nueva materialidad. El aire es pues, también para nosotros, un fluido de variable y diferente viscosidad.

Después de un tiempo, ciertamente cercano, se construye un mapa de arquitecturas que parece haberse sumergido, directa o indirectamente, en esta nueva concepción hidrodinámica del aire. Es posible reconocer otros tipos estructurales y sus correspondientes materialidades aéreas. Es el momento adecuado para promover una investigación rigurosa de las especies de espacios que, guiados por la teoría de Prandtl, surgen como expresión de su estructura. Esta reciente ecuación que une estructura y materialidad espacial permite identificar un mapa de densidades espaciales prescritas en Occidente y construidas en Oriente. 
MEDIATECA DE SENDAI. AIRE LÍQUIDO: ANATOMÍA DE VÓRTICES Noventa años después de la publicación del artículo de Prandtl, Toyo Ito ganó, entre 235 propuestas, el concurso internacional para la construcción de la Mediateca de Sendai. El edificio, situado en el corazón de la ciudad, da habitación a un nuevo programa que combina biblioteca y arte, y en el cual estuvo, finalmente, implicado el propio Ito.

En la propuesta, el arquitecto japonés devela una caja de vidrio, cuyo viejo límite se desvanece en el perímetro que revela el significado de un hueco, el cual es llenado con un aire que se densifica hasta hacerse casi líquido. Lo que caracteriza al proyecto de Sendai son las columnas tubulares (Cortés, 2005), concebidas como algo que se mueve y danza en el agua como algas. El volumen cúbico, que mide $50 \mathrm{~m}$ de ancho y $30 \mathrm{~m}$ de altura, resulta como un tanque de agua.

El orden repetitivo de la estructura de Paxton, inflado de aire newtoniano, es convertido en el proyecto de Ito en trece columnas sinuosas, que parecen dar vida a una nueva especie de aire. Su visibilidad se reafirma en la ausencia de estructuras horizontales. Diseñadas por el ingeniero Mutsuro Sasaki, las vigas son absorbidas en los $40 \mathrm{~cm}$ de espesor que tiene cada forjado. Las trece columnas, pintadas en blanco, son tejidas en finos tubos. Con ellas y su trenzada materialidad, Ito traslada la previsible posición periférica de la estructura hacia su interior, con el objetivo de "diseñar otro tipo de espacio mediante la inserción de estos tubos en un universo espacial... para insertar naturaleza como un elemento, en términos no solo de figura sino también de función, una función orgánica" (Kobayashi y Mulligan, 2002).

Ito concentra todo el material de la capa límite de Prandtl en estas trece columnas metálicas tejidas (fig. 3, 4). El movimiento de la capa límite, desde la periferia hacia su interior, genera un grupo de vórtices aéreos a su alrededor. Su contorneada elevación, que se origina en el plano del suelo y sigue hasta sobrepasar la cubierta, transforma en sus ondulados movimientos el aire previo, inamovible y vacío, en un medio líquido y en constante cambio.

Las trece columnas se convierten en trece vórtices; en elementos estructurales capaces de transformar la materialidad del volumen de aire. Los cuatro vórtices mayores están situados en las esquinas de las plantas, y sirven como principales medios de apoyo y refuerzo. Cinco de los nueve vórtices menores son continuos y dan paso a los ascensores, mientras los otros cuatro son más ondulados y contienen los conductos y el cableado necesario. Witte (2002) elucubra en las columnas de la Mediateca un edificio en el cual la materia -corpórea y programática- es convertida en un complejo de improbabilidades, viscosidades, pulsaciones y remolinos.

De acuerdo a los modelos de Prandtl estos vórtices resultan ser, como flujos de fluidos, muchas veces turbulentos. La velocidad y el tipo de rotación de este aire fluido -identificable en la disposi-

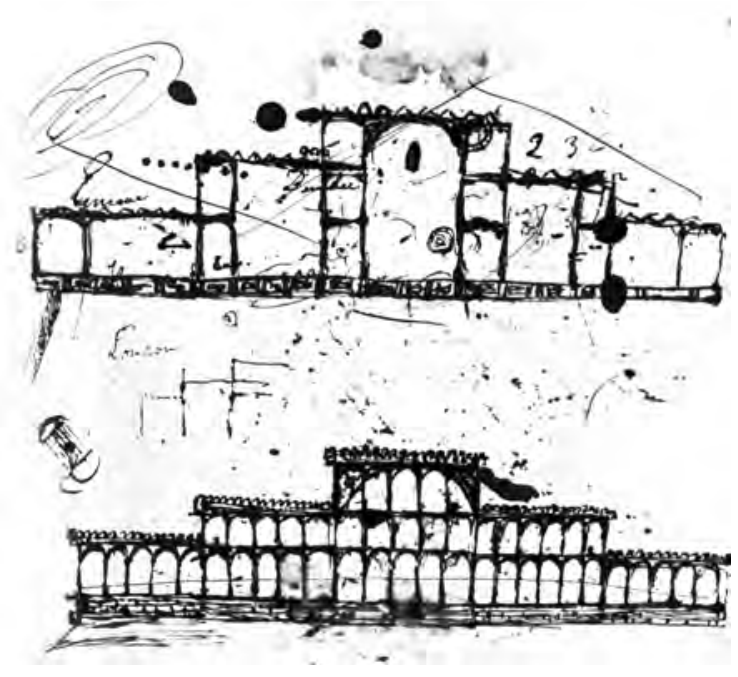

1. Sir Joseph Paxton: Croquis original del Palacio de Cristal.

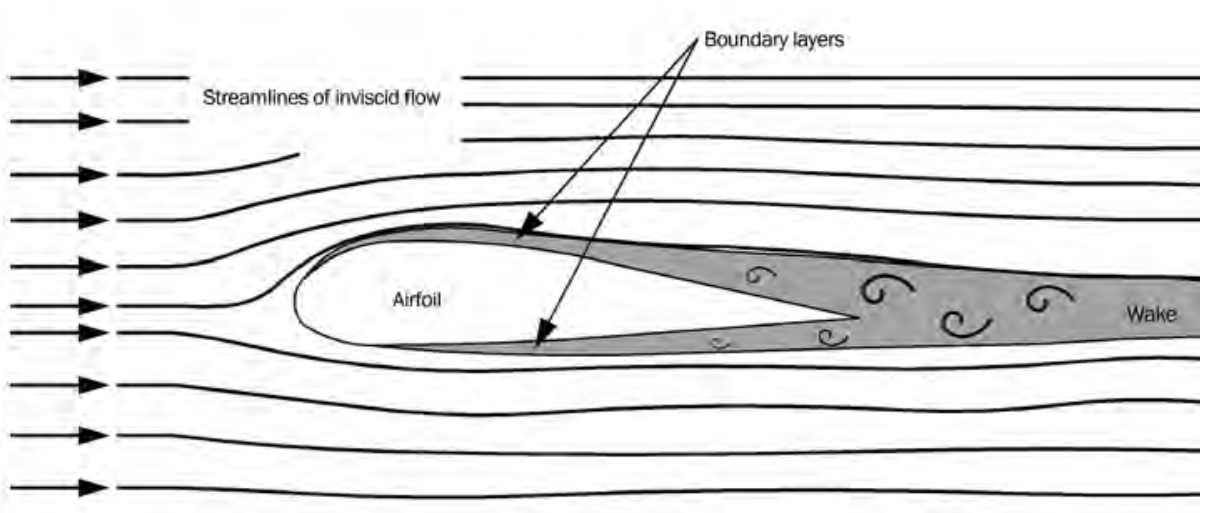

2. Visualización de la teoría de Prandtl, Boundary Layer. El aire como un fluido variable de diferentes capas. 


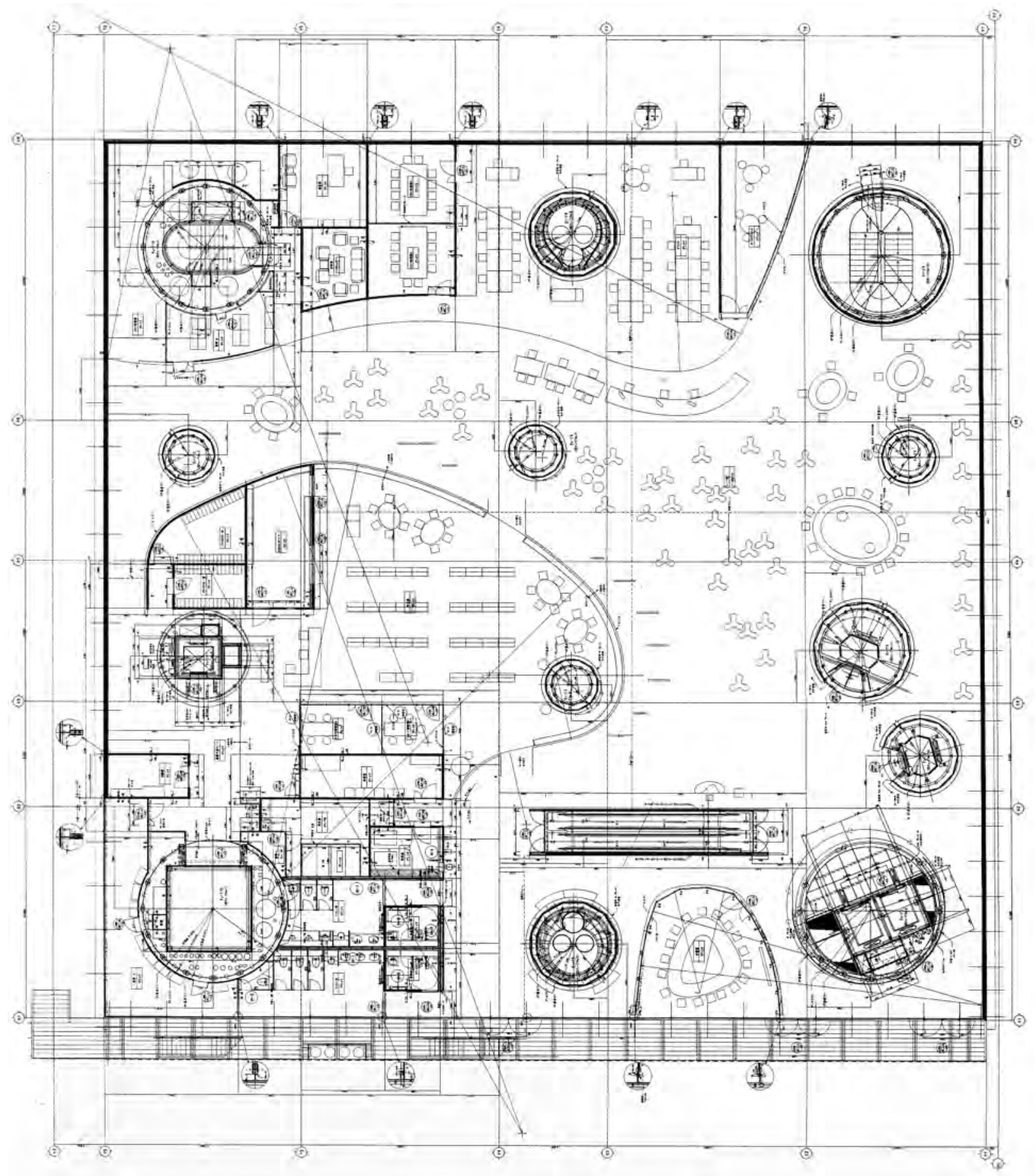

3. Primer piso de la Mediateca de Sendai, Toyo Ito. 

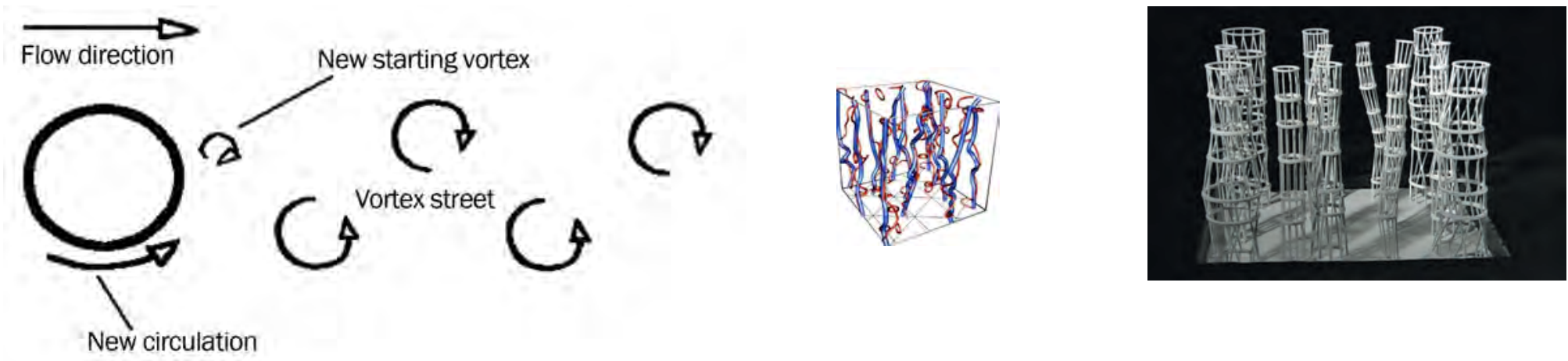

4. Esquema de vórtices: Kármán Vortex Street.

5. Imagen del video de simulación informatizada del baile de vórtices de Smiseth, Smørgrav, Babaev y Sudbø.
6. Maqueta de la estructura de la Mediateca de Sendai. ción geométrica de las luminarias en los techos alrededor de estas columnas-vórtice- son mayores en el centro y decrecen progresivamente con la distancia respecto de él (fig. 5, 6). La ocupación de este nuevo lugar en torno a estos vórtices-columnas sucede en la permanente expectación de ser cambiada por un visitante que resulta ser el "artista de su propio cambio" (Bauman, 2000).

Una investigación paralela en las últimas experiencias sobre dinámica de fluidos, y que manipula gas hidrógeno, son las "Simulaciones Informáticas" de Jo Smiseth, Eivind Smørgrav, Egor Babaev y Asle Sudbø. En ellas transforman el estado gas del hidrógeno a un estado líquido, a través de la manipulación de su viscosidad. Bajo extremos de presión y temperatura, escriben los autores citados, el hidrógeno se convertirá probablemente en un superconductor, llevando la electricidad sin resistencia. Pero además puede, simultáneamente, convertirse en un fluido que fluye sin fricción, un súper fluido. Su video de simulación informatizada nos muestra un baile de vórtices (Lindley, 2005) en el interior de una caja teórica que tiene una increíble similitud con los primeros textos y dibujos del proyecto de Ito.

El color blanco de las columnas en Sendai ilumina en su desplazamiento el interior del vacío, en un viaje que llega hasta descubrir el cielo, para llenarse de una luz que inunda su ausencia de masa. Con las trece columnas el aire transforma su densidad, que llena el tanque de un aire líquido que devuelve a las columnas el movimiento orgánico de las algas. Las columnas sobrepasan el plano de cubierta y, con ella, el nivel líquido, para respirar un cielo inmediato.

La mayor densidad del aire interior marca la diferencia entre el dentro y el fuera, entre el espacio (líquido) y el lugar (gaseoso). El casi inexistente perímetro es solamente visible por la diferente densidad que promueve la nueva anatomía y su líquida ocupación. El mecanismo estructural de Ito es capaz de transformar el aire en un medio líquido, ganando el acceso a una vida acuática para sus habitantes. (Maffei, 2001)

La fachada de vidrio, transformada en frontera liminar, se hace visible como diferencia del aire exterior y el aire interior en esta nueva anatomía. La estructura hace también visible el aire transformado en fluido líquido, antes no visible como simple aire. La anatomía de la Sendai conquista la cualidad fluida que propuso Prandtl en su nueva teoría de las capas límite. El tanque se llena de un aire fluido, casi líquido, como el mundo en el que vivimos. "Nuestro medio físico es ya un medio líquido donde todo fluye y está disuelto", escribe Bauman. La Mediateca tiene la vocación de convertirse en el arquetipo de una nueva arquitectura (Ito, 1997). Un nuevo arquetipo de espacio líquido y fluido.
BIBLIOTECA DE TAMA ART UNIVERSITY. AIRE SÓLIDO: ANATOMÍA MINERAL

En 2005 la Universidad de Tama pidió a Ito el diseño de una nueva biblioteca para su Escuela de Artes, localizada en el campus de Hachioji, en los suburbios de Tokio. Un lugar de bajas colinas ocupadas por anodinos edificios de hormigón y cristal. Ito, que era profesor en Tama, conocía bien este lugar.

Los primeros croquis de Ito se proponían ya abandonar aquella anodina tipología y su también anodina forma de ocupación. Para ello, la primera decisión fue enterrar el edificio, para ofrecer a nivel de suelo un lugar abierto. Ito buscaba un espacio que todos pudiesen cruzar, aunque no tuviesen la intención de ir a la biblioteca. La idea original de un no-edificio pretendía hacer del espacio resultante un lugar abierto e integrado en el campus. Ito quería lograr un nuevo y mejor lugar. Sin embargo, la comisión de la universidad solo buscaba otro edificio de la misma tipología, de tres o cuatro plantas. Pese a ello, Ito no perdió su idea.

Tras aquel desencuentro, su siguiente paso fue elevar su no-edificio sobre el plano del suelo, manteniendo su carácter de bajo-tierra. El segundo proyecto mantuvo la idea de una gran habitación abierta a la calle, a la que sumó la condición genética de un lugar soterrado: una gruta.

Una estructura de cuadrícula curvilínea, en la que se intersectan esbeltos arcos, devela la gruta etérea que Ito andaba buscando. A pesar de tener que trabajar sobre el suelo "nosotros todavía intentamos construir un espacio que recordase una gruta por el tallado del volumen" (Ito, 2007). La idea transfiere a la desnuda estructura la condición de arquitectura en sí misma (fig. 7, 8).

La estructura, que es definida mediante las líneas curvilíneas de esbeltos arcos que arrancan desde los cuatro límites de su forma, se repite a sí misma en forma de nuevas líneas curvilíneas hacia su interior. De esta estructura resulta una gruta, mallada y curvilínea, de arcos de hormigón.

Apliquemos la teoría de Prandtl a la estructura de Tama. La nueva capa límite es ahora su malla curvilínea de arcos. La consideración de esta curvilínea capa límite, como el lugar de máxima viscosidad, multiplica la materialidad alrededor de esta cuadrícula de arcos, multiplicando también la viscosidad del aire previo hasta convertirlo en un cristal fluido: un sólido.

La "Analogía de la Membrana" (Prandtl, 1903) describe una membrana, que se extiende inicialmente y que está curvada de forma perpendicular bajo la presión del fluido. Mohr describe y mide estos esfuerzos y deformaciones a través de geometrías circulares, conocidas como los “Círculos de Mohr”. La expresión geométrica 


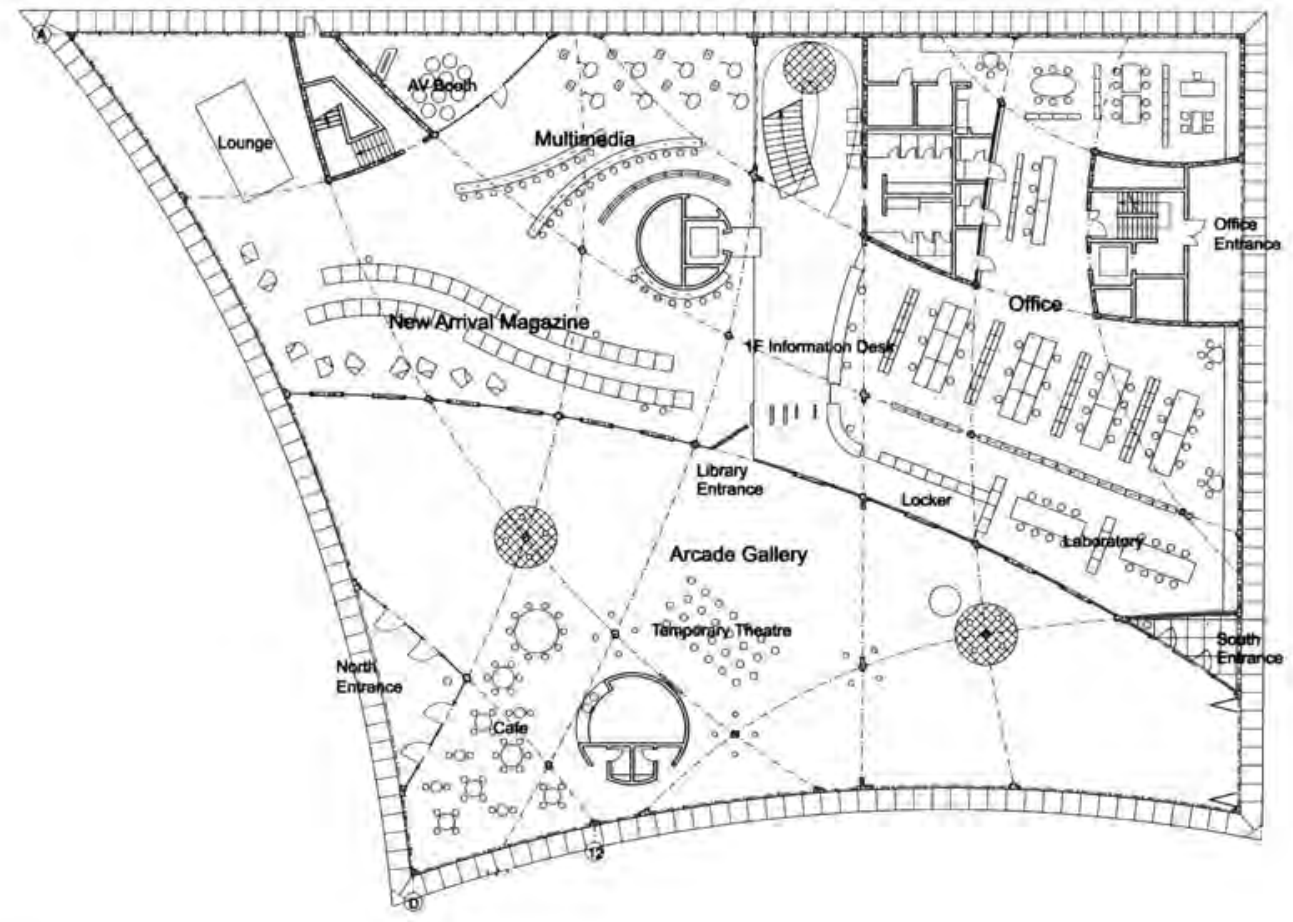

7. Planta primera de la biblioteca en Tama Art University.
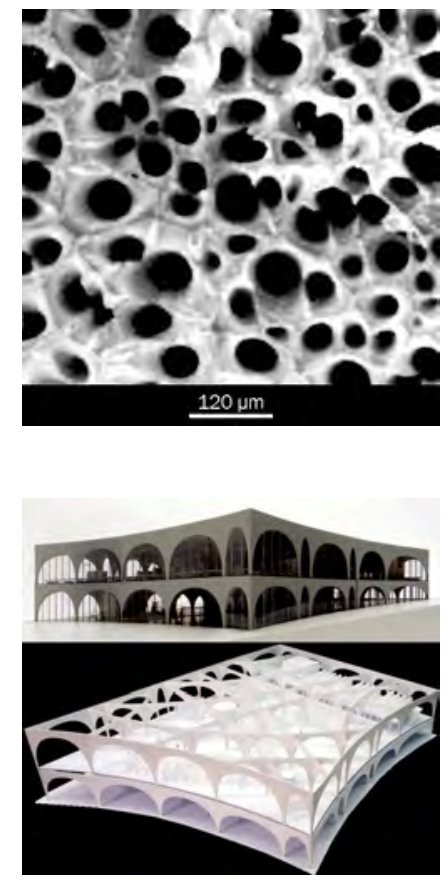

9. Maqueta de la Biblioteca de Tama. de la teoría de la Analogía de la Membrana de Prandtl en fluidos transformados en medios sólidos, resulta de gran similitud a la que aquí se describe.

En este mismo proceso, y en este caso en el Department of Materials, Science and Metallurgy en la Universidad de Cambridge, se compara la estructura de Ito con una operación llamada "Solid-gas eutectic solidification (Gasar) aluminium foam”. En ella, Gergely ensaya la manipulación de una materia metálica, como estructura de un objeto final, y el hidrógeno interior a ella, en estado sólido-gas de punto eutéctico. El proceso lo describe así: "El metal a espumar se funde en un autoclave con una presión controlada de hidrógeno, de modo que la masa fundida se satura con hidrógeno. Esta se solidifica después direccionalmente y, mientras se enfría a través del punto eutéctico sólido-gas, se vuelve sobresaturada. Una doble fase sólido-gas se mezcla simultáneamente -formada a partir de la masa fundida- y se obtiene un sólido poroso anisotrópico con poros cilíndricos orientados en la dirección de solidificación". La materia porosa final y su aire interior, son claramente similares al obtenido por Ito en la biblioteca.

El proceso estructural y constructivo de Tama multiplica el límite estructural en su interior hasta convertirse en una malla. Tal como en el proceso de congelación de un fluido o como en el "Solid-gas eutectic solidification", Ito multiplica la viscosidad del aire alrededor de la estructura de esta nueva malla. Con este nuevo índice de viscosidad, y en una estructura de geometría similar, Ito transforma el gas previo en un fluido cristalino: un fluido sólido.

La mirada, guiada por la ley de Prandt, descubre la biblioteca como un lugar de mineralización etérea. La gruta lograda sobre el suelo ofrece la visión de dos tipos de aire muy diferentes. El aire a su alrededor es simplemente aire, el aire del campus de Tama Art University. El aire interior a la gruta, el aire que rodea y envuelve la estructura desnuda, es un aire fluido y sólido. De la cuadrícula curvilínea de arcos emerge un aire de máxima viscosidad, de naturaleza casi mineral.

Interior y exterior, y sus diferentes tipos de aires, expresan dos tiempos diferentes. En el derredor del edificio el tiempo se mueve en un día donde suceden las 24 horas. En las entrañas de la malla curvilínea el tiempo de este otro aire parece parar en otro tiempo, que vive por un instante, en un tiempo también mineral.

La relación de su dentro y fuera, en términos del movimiento moderno, ya no es una cuestión de fronteras o límites. Aquí, la relación ha sido transformada en términos de materialización de aire. En el fuera que rodea la biblioteca el aire posee una densidad menor que en el aire que hay interior a aquella anatomía. La relación entre fuera y dentro es producida, pues, por una diferencia de densidad y materialización de este aire. Fuera el aire es aire, solo aire. Dentro el aire es un fluido-sólido. El lugar es gas, el espacio es sólido.

La estructura, concebida como anatomía real de este nuevo aire, resulta ser quien lo transforma en un volumen de fluido-sólido. En la visión de la estructura vemos el volumen de aire. A través de la estructura emerge el aire como la materia esencial de esta nueva arquitectura. La estructura transforma y permite reconocer esta nueva especie de aire sólido. En Tama Art University, escribe el equipo editorial de la revista Domus en 2007, Toyo Ito anuncia un nuevo modo estructural: estructuras acrobáticas donde él ha reconocido su idea de arquitectura como una etérea caverna (fig. 9). 


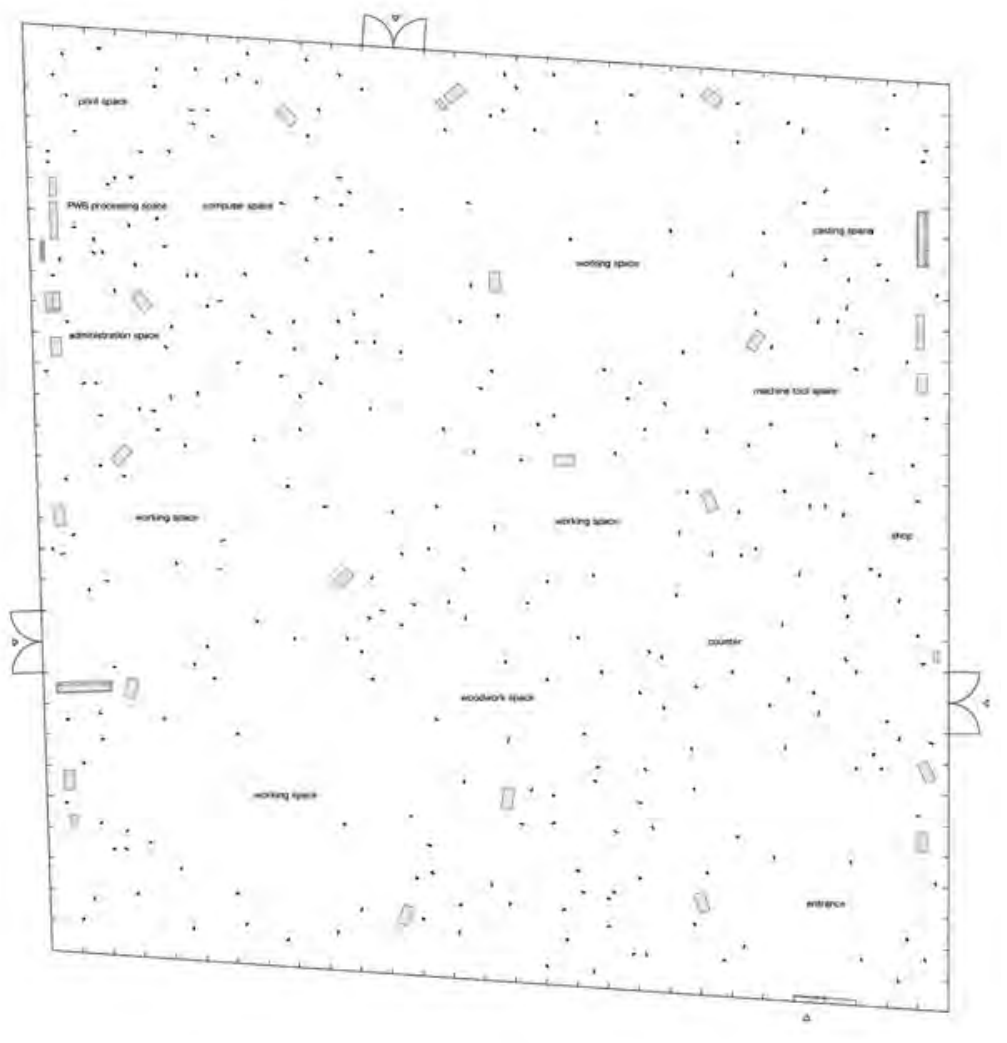

10. Plano del Kanagawa Institute of Technology KAIT, Junya Ishigami.

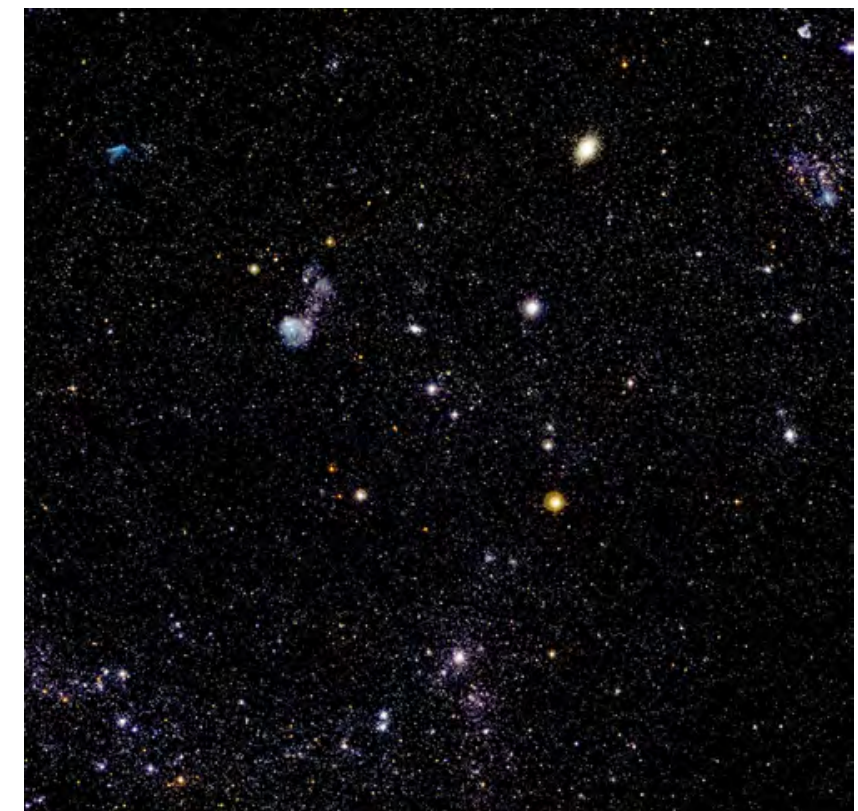

11. Imagen de la Nube de Magallanes.
La transparencia de este fluido es lograda en el contraste con el aire previo. Entrar en la biblioteca significa dar el paso que abandona un lugar hecho de aire gaseoso para sumergirse en uno nuevo de materia sólida. Esta operación transformadora del aire ya no necesita de la modificación geográfica de la primera propuesta. La planta de suelo de la biblioteca evita, incluso, modificar la pendiente del lugar, sometiéndose a ella. Ito solo trabaja ya con aire.

La mineralización de este aire, a través de la multiplicación de la cuadrícula curvilínea hacia su interior, ofrece al visitante el tiempo de habitación de sus concavidades cristalizadas. Simultáneamente, ofrece también un movimiento que fluye en este medio de fluido-sólido. Una razón por la cual Ito apela al concepto de cueva es su cualidad íntima que equilibra la articulación espacial y la continuidad (Pollock, 2008). Para trasladar esta metáfora a un vocabulario arquitectónico, Ito traza una geología de arcos de reminiscentes estalactitas.

Esta concepción espacial da libertad y riqueza a la arquitectura (Ito, 2007), recibe la energía natural que emana de la tierra y la transforma en orden arquitectónico. Su habitante es un hombre que modifica su tiempo en un tiempo contemporáneo que probablemente no logra fuera de esta anatomía. Ellos son hombres y mujeres que habitan nuevas especies de espacios: espacios de fluidos sólidos.

\section{KAIT. AIRE PLASMÁtico: ANATOMÍA DE ELECTRODOS}

Parece posible leer en la nube plasmática de Magallanes los primeros croquis y dibujos de Junya Ishigami para el Kanagawa Institute of Technology KAIт. El mapa de estrellas alrededor de la nube de gas resulta familiar a la nube de pilares metálicos que envuelven de forma aleatoria lugares vacíos. Los puntos estructurales del KAIT parecen traducir aquellas estrellas de la nube de Magallanes. El estado de su aire, en permanente interacción, parece coherente a esta escuela de viajeros de ida y vuelta localizada en Atsugi, un suburbio a $32 \mathrm{~km}$ al oeste de Tokio.

La arquitectura del KAIT, entendida como una operación experimental, nos sumerge en el sistema de trabajo del científico que explora en el proceso de formación de un plasma artificial. No en vano, este es un edificio para proveer espacio para la experimentación extracurricular. Y así Ishigami explora sobre espacio y materia, y convierte el proyecto también en un lugar de experimentación.

El plasma artificial es generado cuando una corriente eléctrica atraviesa un fluido. Su diferencia de potencial provoca un campo eléctrico que lleva los electrones (negativo) hacia el ánodo (electrodo positivo), mientras que el cátodo (electrodo negativo) los lleva hacia el núcleo. La planta del KAIT, siguiendo este proceso eléctrico, transforma la habitación-edificio en un laboratorio-contenedor de aire de $2.268 \mathrm{~m}^{2}$. Forbet reclama para esta habitación-edificio -y para otras como ella-, a través de la visión de las pinturas de Vilhelm Hammersh $\varnothing$ i (Forbet, 2008), un espacio interior que da valor a un muro (nuestra capa límite)... y a un volumen de aire, demostrando el significado de "la habitación" como el elemento primario de la arquitectura. Habitación es aquí un laboratorio real de aire.

Ishigami y su ingeniero Yasutaka Konishi introducen una amalgama de 305 puntos en este contenedor rectangular de aire. La posición de estos puntos es indefinible: 305 columnas distribuidas de forma aparentemente aleatoria y con formas también aparentemente arbitrarias. Como en la nube de Magallanes, no somos capa- 

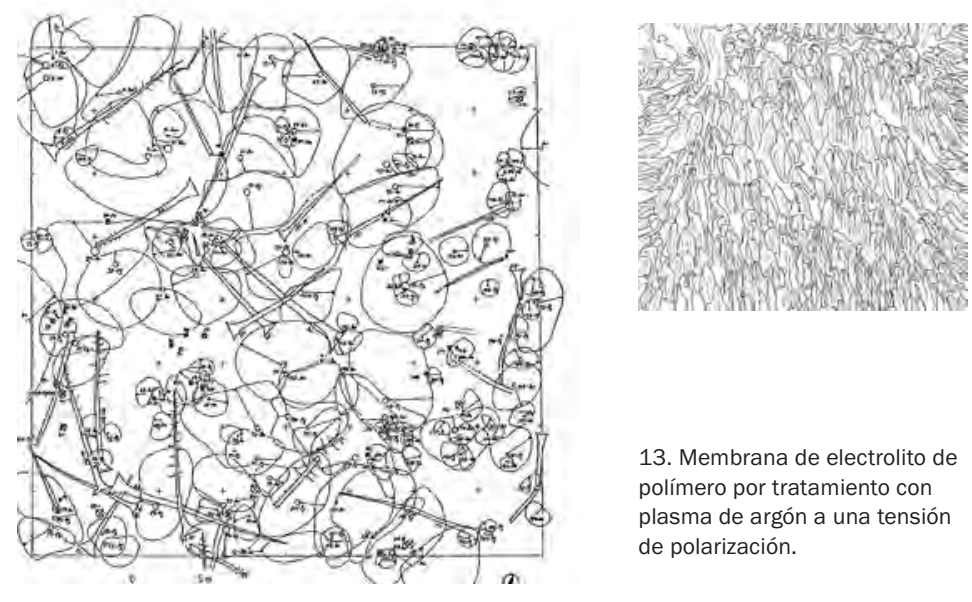

13. Membrana de electrolito de polímero por tratamiento con plasma de argón a una tensión de polarización.

12. Proyección de cubierta - árboles, Junya Ishigami.

ces de identificar ley alguna. $\mathrm{Y}$ es que, como el mismo autor confirma, pretende crear un edificio donde no es claro si hay reglas. Ishigami y Konishi continúan al proceso de inserción de estos 305 puntos una etapa diferente. Introducen un nuevo elemento a la habitación-laboratorio: una cubierta metálica. El peso y distribución de esta estresa la situación de carga de los pilares metálicos, de la misma manera que lo hace la corriente eléctrica en el proceso artificial plasmático.

Los 305 pilares son aparentemente iguales, como los electrones. Sin embargo, son diseñados para tener diferentes formas de trabajo en su relación con aquellas nuevas cargas. Como los electrones -negativos o positivos-, los pilares trabajan de forma opuesta: a compresión o a tracción (fig. 10, 11). Aunque sus formas son similares, los pilares trabajan de diferente forma. En el mapa de pilares, Ishigami indica que hay 42 unidades que trabajan a compresión para atender las cargas verticales, y 263 postensados que asumen cargas horizontales trabajando como muros cubierta. Unos y otros están impresos en blanco, como las estrellas de Magallanes, y sus formas transitan desde las más finas y tensionadas, que van de 16 a 14,5 mm, a las más gruesas y comprimidas, que miden entre 63 y $90 \mathrm{~mm}$.

Como la corriente eléctrica estabiliza la materia por polarización, en la habitación experimental del KAIT se logra por equilibrio de cargas. En este mismo sentido, Sumner define el espacio del KAIT como de "polos opuestos" (Sumner, 2008).

Con el fin de alcanzar este equilibrio, los encargados de su montaje -entendidos como el elemento conductor del proceso- sitúan los elementos comprimidos y la estructura de la cubierta en su

lugar (Pollock, 2008). Cuelgan de las vigas los elementos en tensión, sin tocar el suelo, hasta no introducir las cargas simuladas de peso de nieve. Cuando las cargas son eliminadas, el acero tensado encuentra su lugar y la cubierta aparece como se esperaba. La anatomía de este nuevo volumen de aire fluido es polarizada en un nuevo equilibrio de columnas comprimidas (electrones negativos) y columnas tensionadas (electrones positivos). Esta idea de equilibrio es descrita por la editorial de The Architectural Review en sus Premios AR (Finch, 2008) como un equilibrio de orden visual, que considera el concepto de delgadez y la visión diagonal de dichos elementos.

En el proceso eléctrico este estado intermedio se llama "avalancha de ionización”. En él, la colisión entre electrones y átomos del gas neutro forma más iones y electrones. Con suficiente corriente el fluido es paulatinamente convertido, en su densidad e ionización, en un equilibrio térmico llamado plasma.

El nuevo espacio logrado no es un espacio universal regulado por una malla de columnas dispuestas entre el plano del suelo y el cielo raso de la cubierta. En esta habitación-laboratorio Ishigami crea un espacio particular -un universo propio- modificando su aire a través de una nueva estructura, la anatomía de su particular universo. El resultado es un fluido de estado plasmático, cuya interacción eléctrica resulta ser la relación de equilibrio entre los pilares comprimidos y los traccionados. Entre estos pilares se descubren las corrientes de un equilibrio eléctrico, que surgen desde las cuatro entradas y cuyo discurrir origina diez lugares vacíos. Los recorridos son caminos informales, y sus lugares están hechos para una variedad de funciones que ocupan los claros. Su itinerar desvela el estado variable de un aire-fluido a través de la estructura, su anatomía. El movimiento del observador produce con las columnas cortinas de variable densidad en su yuxtaposición, activando la inestabilidad propia de la materia plasmática.

De acuerdo a la teoría de Prandtl, el material de la capa límite ha sido desmaterializado en estos 305 puntos, casi ya electrones. El máximo nivel de excitación y viscosidad se traslada alrededor de esta amalgama de puntos y, con ellos, se extiende de forma plasmática al resto del volumen de aire de esta habitación-laboratorio. La disolución de esta estructura, de materialidad no estable, se mueve con el equilibrio de polos opuestos, que señalaba Summer. Esta permanente acción de búsqueda de un nuevo equilibrio determina la forma de habitación de aquellos estudiantes de ida y vuelta y la inestable materialidad de su aire y de su vida (fig. 12, 13, 14). 
Esta investigación propone, de acuerdo

a la teoría de Prandtl, la manipulación de

este aire considerándolo como un fluido

de diferentes capas y viscosidades. La

manipulación del aire como espacio, a través

de una nueva estructura que se asimila a

la capa límite de Prandtl, logra definir las

diferentes materialidades de este nuevo aire.

La estructura, su anatomía, es la forma por la

cual somos capaces de hacer este aire visible, que emerge como nuevas especies de aire.

LA ANATOMÍA DEL AIRE: NUEVAS ESPECIES DE ESPACIOS

La arquitectura que heredó el movimiento moderno suponía muchas veces la construcción de un gran envoltorio. Su interior era destinado, en términos físicos, a envolver un volumen de aire; aire y nada más. El interior, como tal, era un lugar vacío con vocación de ser ocupado.

El Palacio de Cristal de Paxton muestra, a través de su estructura, la evidencia de este volumen de aire de materialidad newtoniana. El aire es un volumen vacío que espera ser ocupado, no manipulado.

Esta investigación propone, de acuerdo a la teoría de Prandtl, la manipulación de este aire considerándolo como un fluido de diferentes capas y viscosidades. La manipulación del aire como espacio, a través de una nueva estructura que se asimila a la capa límite de Prandtl, logra definir las diferentes materialidades de este nuevo aire. La estructura, su anatomía, es la forma por la cual somos capaces de hacer este aire visible, que emerge como nuevas especies de aire. Estas nuevas especies de aires son, para nosotros, nuevas especies de espacios.

Asimismo, el proceso de logro de nuevas especies de espacios, como resultado de la manipulación de las anatomías del aire que lo forman, ha llevado a los arquitectos de Oriente a generar laboratorios espaciales que buscan novedosas tipologías estructurales. El laboratorio doméstico de la modernidad se ha desplazado al labora- torio de una nueva arquitectura pública, para una nueva contemporaneidad que ahora une Oriente y Occidente. La estructura de este nuevo aire ha emergido en estos laboratorios en nuevas y diferentes anatomías. En ellos, la manipulación de la estructura otorga al aire su propia anatomía, y con ella un nueva y propia densidad.

El aire es ahora un material visible que ocupa y materializa su propia anatomía. El aire y su anatomía es un espacio en sí mismo. En estas nuevas anatomías de aire descubrimos las nuevas especies de espacios de nuestra contemporaneidad.

Estas nuevas anatomías de Oriente, que consideran la cualidad fluida del aire de acuerdo a la teoría de Prandtl, han vuelto a Occidente para descubrir con el filósofo polaco Zygmunt Bauman un nuevo habitante de estas nuevas especies de espacios. Ellos son hombres y mujeres en cuya vida todo fluye, como escribe Bauman, viviendo una nueva "modernidad líquida". ARQ

Javier Pérez-Herreras | Arquitecto y Doctor en Arquitectura, Universidad de Navarra, 2009. En 2002 funda, en conjunto con Javier Quintana de Uña, el Taller Básico de Arquitectura, un laboratorio de investigación con sedes en Madrid y Pamplona. Su trabajo profesional y como investigador ha obtenido numerosos premios y reconocimientos y ha sido expuesto en Venecia, Bruselas, Nueva York y Madrid. Entre 2005 y 2009 fue rector de la nueva Universidad San Jorge de Aragón; actualmente es profesor titular de Proyectos arquitectónicos en el Departamento de Arquitectura de la Universidad de Zaragoza.

Bibliografía

BAUMAN, Zygmunt. Liquid Modernity. Blackwell Publishing Ltd., Cambridge, 2000.

CORTÉS, Juan Antonio. "Beyond Modernism, Beyond Sendai". Revista El croquis № 123. Editorial El croquis, Madrid, 2005, p. 21.

FINCH, Paul (ed.). "AR Awards for Emerging Architecture 2008". Revista The Architectural Review No 1342. EMAP Construct, Londres, diciembre de 2008, p. 56.

FOBERT, Jamie. "The Great Indoors". Revista The Architectural Review № 1339. EMAP Construct, Londres, septiembre de 2008 , p. 49

GERGELY, Vladimir. "Solid-gas eutectic solidification (Gasar) aluminium foam". DolTPoMS,

University of Cambridge, Cambridge, 2002. Disponible en http://core.materials.ac.uk/.

[Revisado el 14 de april de 2012].

ITO, Toyo. “Mediateca de Sendai”. Revista 2G No 2. Editorial Gustavo Gili, Barcelona, 1997, p. 26 ITO, Toyo. “The Spider's Web". Revista Domus № 906. Editoriale Domus, Milán, septiembre de 2007, p. 37.

KOBAYASHI Hiroto y Mark MULLIGAN (trad.) "Mediatheque Project Committee meeting minutes, first meeting August 28, 1995". Toyo Ito Sendai Mediatheque. Harvard Design School - Prestel, Nueva York, 2002.

LINDLEY, David. “Two Quantum States for the Price of One”. Physical Review Letters No 16, 2005 Disponible en http://prl.aps.org/. [Revisado el 14 de april de 2012].

MAFFEI, Andrea. "Toyo Ito. Le opere, i progetti, gli scritti. Tre Trasparenze, Trasparenza fluida". Revista Casabella № 693. Electa, Milán, 2001, p. 83, 346.

POLLOCK, Naomi. "Kanagawa Institute of Technology Workshop". Revista Architectural Record Vol 196 No 11. McGraw Hill Construction, Nueva York, noviembre de 2008, p. 129.

PRANDTL, Ludwig. "Zur torsion von prismatischen stäben". Physik Zeitschrift 4, 1903, p. 758-770. PRANDTL, Ludwig. Resistance of Bodies. In Essentials of Fluid Dynamics. Blackie \& Son Limited, Londres y Glasgow, 1952, p. 175.

ROCHON, Lisa. "Catching air". Air. Massachusetts Institute of Technology, Cambridge, 2010, p. 41. SMISETH, Jo; SMøRGRAV, Eivind; BABAEV, Egor y Asle SUDBø. "Observability of a Projected New State of matter: A Metallic Superfluid". Physical Review Letters № 95. Disponible en http://prl. aps.org/. [Revisado el 14 de april de 2012].

SUMNER, Yuki. “Poles Apart”. Revista The Architectural Review No 1339. EMAP Construct, Londres, septiembre de 2008, p. 61.

WITTE, Ron. "Solution". Toyo Ito Sendai Mediatheque. Harvard Design School - Prestel, Nueva York, 2002, p. 20. 